\title{
Bilateral simultaneous total hip arthroplasty: comparison of treatment outcome between the direct anterior approach and the posterolateral approach
}

\section{Shuo Feng}

Affiliated Hospital of Xuzhou Medical University

Ning Jian Sun

Affiliated Hospital of Xuzhou Medical University

Yu Zhang

Affiliated Hospital of Xuzhou Medical University

\section{Ye Zhang}

Affiliated Hospital of Xuzhou Medical University

\section{Yang Xiang Chen}

Affiliated Hospital of Xuzhou Medical University

Guo-Chun Zha ( $\nabla$ xzcgcgk@163.com )

Affiliated Hospital of Xuzhou Medical University

\section{Research article}

Keywords: Hip replacement, Hip joint, DAA, PLA, Simultaneous, Bilateral

Posted Date: November 7th, 2019

DOI: https://doi.org/10.21203/rs.2.16783/v1

License: (c) (i) This work is licensed under a Creative Commons Attribution 4.0 International License.

Read Full License 


\section{Abstract}

Background There are many surgical approaches for total hip arthroplasty. In recent years, direct anterior approach (DAA) has been highly praised by many scholars, and it has been widely reported that it has a good curative effect, such as fast recovery. Whether the surgical results and patient satisfaction can reach or exceed the traditional posterolateral approach has been controversial. We hypothesized that the treatment outcome of the direct anterior approach (DAA) approach is superior to the traditional posterolateral approach (PLA). Methods From January 2015 to April 2017, 20 patients (40 hips) were randomly divided into posterolateral approach (PLA) group and direct anterior approach (DAA) group. Record the operation time on both sides, postoperative drainage, prosthesis position, and complications, the functional recovery of hip joint was evaluated by D'Aubigne-Postel score, and the postoperative pain was evaluated by VAS score. Gait parameters were measured before surgery and at 3 and 6 months after surgery, and the patients were asked which side they preferred subjectively after surgery. Results When compared with the PLA group, the DAA group had a shorter incision length (11.4 vs $14.72 \mathrm{~cm}, \mathrm{P}<0.001)$, shorter intraoperative blood loss (184.05vs $219.00 \mathrm{~mL}, \mathrm{P}<0.001)$, shorter postoperative drainage volume (105.35 vs $154.10 \mathrm{~mL}, \mathrm{P}<0.001$ ), and lower VAS scores. However, the PLA had shorter operative times (82.20 vs $67.3 \mathrm{~min}, \mathrm{P}<0.001)$. There was no significant difference in acetabular inclination (39.72 vs $\left.40.92^{\circ}, \mathrm{P}=0.069\right)$, and acetabular anteversion $\left(17.41 \mathrm{vs} 17.69^{\circ}, \mathrm{P}=0.663\right)$ between the two groups. Joint function recovery: The D'Aubigne-Postel scores of the hip joints in the DAA group vs PLA group at 1, 3, and 6 months after surgery were $(8.2,11.5,16.5)$ vs $(7.65,11.45,16.9)$. The difference in the 1,3 month was statistically significant $(P=0.012, P=0.038)$, however, this difference disappeared in the 6th month $(P=0.072)$. Conclusions Compared with the PLA, the DAA has the advantages of shorter operation time, smaller incision, less bleeding, less pain, better gait performance and faster recovery of joint function, but patients subjectively preferred the traditional PLA.

\section{Background}

Total hip arthroplasty (THA) is an effective method to treat end-stage diseases of hip joint, reducing pain, improving joint function and quality of life[1, 2]. However, there are still some patients with THA who still have joint pain, walking instability and other problems after surgery. These problems limit the recovery of hip function. Poor placement of the prosthesis, instability of the prosthesis, soft tissue damage, etc. may be the cause of these problems. Choosing the best surgical approach can minimize these risks and further improving the efficacy of total hip arthroplasty.

The National Joint Registry of England date showed most surgeons prefer posterior surgery, followed by lateral surgery. $59 \%$ of surgeons[3] choose posterolateral approach (PLA) in THA, this approach usually requires cutting the short external rotation muscle group and the joint capsule, which will damage the posterolateral structure, resulting in weak posterior soft tissue. Therefore, some scholars believe that the posterolateral approach (PLA) not only increases the risk of dislocation during hip flexion, adduction, and rotation, but also has a larger surgical trauma and a longer recovery time of hip function than direct anterior approach (DAA)[4]. The direct anterior approach (DAA) has been widely discussed and reported 
in various magazines and meetings in recent 10 years. According to statistics, nearly $20 \%$ of total hip arthroplasty in the United States uses this approach. Proponents believe that the DAA approach is a direct muscle gap approach, does not cut any muscles and cause little damage to the abductor muscles, can better preserves soft tissue integrity, and achieves good surgical results[5-7].

Some scholars have reported that compared with the traditional posterolateral approach, patients with DAA approach improved joint functional during the first 6 weeks to 6 months[6]. However, Meneghini[8] showed a similar overall muscle injury to DAA and PLA in a cadaveric study, and the DAA approach had less damage to the gluteus minimus (DAA averaged $8 \%$, PLA averaged 18\%). However, tensor fascia lata and rectus femoris have a heavier damage in the DAA approach (DAA averaged 31\%, PLA averaged 12\%). $50 \%$ of the piriformis or conjoined tendons will be cut off in $50 \%$ of the DAA approach, while in the PLA approach, the abductor muscles will be completely cut off and then repaired and sutured. Graves[9] and Rathod[10] found that DAA is similar to the expected outcome of PLA, and any benefit from DAA patients is short-lived and modest. Many people ignore the complications of the wound, the injury of the cutaneous nerve, the fracture around the prosthesis and so on. DAA is technically demanding and takes a long time to learn, many authors report a steep learning curve[3,5]. Compared with the DAA, the patients subjectively prefer the PLA[11].

In most studies, THA is usually performed on one side, and patients are divided into two groups by surgical approach, different surgery doctor, different preoperative and post-operative care schemes will result in bias. Few scholars have used gait analysis data with objective and detailed kinematics to compare the results of DAA and PLA. In view of this, we designed a simultaneous bilateral THA for patients, DAA on one side and PLA on the other side, the operation was performed by the same doctor. The differences in clinical outcomes and subjective satisfaction were compared, and gait analysis was used to evaluate joint function after THA. We would like to emphasize that, as far as we know, this is the first study to compare the differences in efficacy between DAA and PLA from three aspects: doctors, patients, and objective kinematic data. We hypothesized that DAA will show better efficacy, higher patient satisfaction, and better joint function.

\section{Methods}

\section{Patients}

This study is a prospective randomized controlled case study and used blind methods. The evaluator is trained and does not know the specific test plan. This study involved human participants and all procedures performed in the study were in accordance with the Medical Institution Administration Regulation, conformity with the ethical standards of the National Research Council and Helsinki Declaration. This study was approved by the Ethics Committee of Affiliated Hospital of Xuzhou Medical University, registration number (XYEY2014-xjs010-02).

All patients undergoing primary THA at our hospital from January 2015 to April 2017. All patients received the same THA care, pain management, and rehabilitation program before and after surgery. Gait 
analysis was performed at the gait analysis laboratory of Xuzhou Medical University.

Inclusion criteria:(1)Primary bilateral simultaneous THA;(2) Implanted with the same design and type of uncemented prosthesis on both sides;(3) Hip osteoarthritis;(4) Avascular necrosis of femoral head;(5) DDH (Crowe Type 1 or2);(6) Informed consent, signed informed consent, and agreement to participate in clinical research;(7) Same surgeon.

Exclusion criteria;(1) Severe internal medical disease comorbidities;(2) Severe hip anatomic deformity;(3) History of hip infection;(4) Ipsilateral lower extremity deformity;(5) Diseases in other ipsilateral joints;(6) Neurological or musculoskeletal diseases affecting gait;(7) Reluctant to join this clinical research.

After the evaluation, 37 patients were eligible for this study, 15 patients refused to join the study, and 2 patients were lost for follow-up. A total of 20 patients were included in the study, 9 males and 11 females with an average age of 63.0 years old (range 51-73). Patients were randomly assigned before surgery and sealed opaque envelopes were created using computer generated random lists. (Fig1)

\section{Surgical procedures}

All patients underwent general anesthesia. All operations were performed by the same surgeon in the same hospital, and the surgeon was specially trained and experienced in both approaches. And before the study, the doctor went far beyond the learning curve and completed hundreds of DAA and PLA total hip arthroplasty independently.

DAATHAs take the lateral decubitus position, starting from $2 \mathrm{~cm}$ below and outside of the anterior superior iliac spine, and making a surgical incision about $10 \mathrm{~cm}$ long to the distal end. The fascia of the skin, subcutaneous, and fascia lata is cut open layer by layer. The outer cutaneous nerve is protected and the lateral branch of the circumflex femoral artery is ligated. The fat pad above the joint capsule was removed, the joint capsule was fully exposed, the front hip joint capsule was removed, and the femoral neck was revealed. The femoral neck was amputated and the femoral head was removed. Excision of the acetabular peripheral hyperplasia and foreign body, and then gradually increase the diameter of the acetabulum to polish the acetabulum, control the proper anteversion and inclination angle, and increase the diameter of the acetabulum to the appropriate size., install the cup, put the acetabular lining. The hip joint is externally rotated and extended, and the proximal end of the femur is raised to expand the medullary cavity. The femoral stem prosthesis is inserted into the medullary cavity and pressed tightly, and then the femoral head is mounted. Reset the hip joint, check hip stability, and compare the length of both lower limbs. Place a drainage and suture the wound.

PLATHAs take the lateral decubitus position. Taking the apex of the great trochanter as the center point, making an arc incision about $13 \mathrm{~cm}$ in length. The skin, subcutaneous tissue, and fascia lata were cut layer by layer, and the external rotation muscle group was exposed and separated. The posterior joint capsule was exposed and cut, the femoral neck was exposed, the femoral neck was cut, and the femoral head was taken out. Excision of the acetabular peripheral hyperplasia and foreign body, and then 
gradually increase the diameter of the acetabulum to polish the acetabulum, control the proper anteversion and inclination angle, and increase the diameter of the acetabulum to the appropriate size., install the cup, put the acetabular lining. Exposing the proximal femur and expanding the medullary cavity. The femoral stem prosthesis is inserted into the medullary cavity and pressed tightly, and then the femoral head is mounted. Reset the hip joint, check hip stability, and compare the length of both lower limbs. Place a drainage and suture the wound.

\section{Rehabilitation}

All patients were treated with the same nutritional intervention, antibiotics, thrombosis prevention, perioperative pain management, and the same nursing and rehabilitation exercise program. One hour before the operation, all patients received cefazolin according to body weight. Postoperative analgesia was administered with $200 \mathrm{mg}$ celecoxib orally. Drainage tubes were not attached in incision. The wound dressing change was carried out by the same doctor, and the patients were guided to walk with the walker after the operation, and informed of the relevant precautions and the standard preventive measures to prevent dislocation. Blood transfusion was determined according to the clinical manifestation and blood routine test results, such as patients with obvious anemia symptoms or hemoglobin below $70 \mathrm{~g} / \mathrm{L}$ will be given blood transfusion. All patients started subcutaneous injection of low molecular weight heparin 12 hours after surgery (4100IU, qd, Clexane, Sanofi-Aventis, France). Continued oral rivaroxaban after discharge (5mg, qd, Xarelto, Bayer, Cologne, Germany). To prevent deep venous thrombosis of lower limbs, the minimum time of anticoagulation is 5 weeks. Standard rehabilitation programs, including weight-bearing and assisted walking, were implemented the next day after surgery. Hip flexion reaches $100^{\circ}$ flexion and $40^{\circ}$ abduction and can walk independently and safely, the patients can leave the hospital.

\section{Assessment}

The preoperative and postoperative data of the patients were collected by the graduate students, and the intraoperative data were collected according to the surgical records. Hip joint X-ray for imaging evaluation (including position and stability of prosthesis) and the patients were reviewed in the outpatient according to the routine THA review program (first review is in the sixth week and every three months thereafter). D'Aubigne - Postel score for hip function assessment. VAS score for pain assessment.

At the same time, all patients were asked to answer the same questions, these questions were not included in the D'Aubigne - Postel score[12]. Ask the patient which side is better and why (provide explanation if possible)? If they have to undergo total hip arthroplasty, which method they would recommend.

Gait analysis was performed before and 3 and 6 months after surgery. All gait analyses were performed by the same professional two technicians, who did not participate in patient care and were unaware of the clinical information of all patients. According to gait analysis protocol囚a total of 29 markers are 
attached to the bilateral lower extremity bony markers( including anterior superior iliac spine, posterior superior iliac spine, sacral, thigh, knee, tibia, heel, ankle, toe ) $[13,14]$.

\section{Statistical analysis}

Statistical data were processed using SPSS 23.0 statistical software and a threshold of significance of $p$ $<0.05$. Results were reported as mean $\pm \mathrm{SD}(X \pm S)$. Demographic and clinical data were compared between the DAA and PLA groups using Pearson's chi-squared test. Inter-group differences were assessed for significance using independent $\boldsymbol{t}$ tests after confirming that the data followed a normal distribution.

\section{Results}

A total of 20 patients were included, no loss during the follow-up period. There were 9 males and 11 females, with an average age of 63.00 years. All cases were bilateral hip joint disease, 4 cases of osteoarthritis, 2 cases of DDH (One was in stage II of Crow's classification and the other was stage I), and the remaining 14 cases were avascular necrosis of the femoral head.

Compared with PLA, DAA had a longer operation time (about 15 minutes on average, $\mathrm{P}<0.05$ ), but intraoperative bleeding (average about $35 \mathrm{~mL}, \mathrm{P}<0.05$ ) and surgical incision length (average about 3.6 $\mathrm{cm}, \mathrm{P}<0.05$ ) were less than the PLA group.

Postoperative X-ray showed that there was no significant difference in acetabular inclination (39.72 vs $\left.40.92^{\circ}, P>0.05\right)$, and acetabular anteversion ( 17.41 vs $\left.17.69^{\circ}, P>0.05\right)$ between DAA and PLA (Fig2). At the last X-ray review, there was no acetabular loosening change in the DAA and PLA groups, and both groups had bone ingrowth in the acetabular and femoral prostheses.

One intraoperative complication occurred in the DAA group (non-displaced trochanteric fracture), while intraoperative complications were not recorded in the PLA. There was no significant difference in the incidence of postoperative complications between the two groups during the 6-month follow-up. Only 2 patients developed postoperative complications. Including a patient developed an incision hematoma in the PLA group, which improved without treatment. One of the patients in the DAA group had a poorly aligned incision at the incision, which improved after healing and was not associated with superficial infection. No revision in either group.

On the 1st, 3rd, and 5th days after surgery, the VAS score of the DAA side was lower than that of the PLA side $(P<0.05)$, but the difference disappeared on the 7th day. DAA had a higher D'Aubigne-Post score at 1 st and 3rd months after surgery $(P<0.05)$, but the difference was not significant at 6 th month after surgery $(P>0.05)$. Similarly, several gait parameters were also significantly different between the two groups at the 3rd month after surgery $(p<0.05)$, but these differences disappeared at the 6th month $(p>$ 0.05) (Fig3).

From the patient's feelings, 13 of them (65\%) prefer the PLA side and 7 (35\%) prefer the DAA side. The explanations they gave are shown in Table 1. 


\section{Discussion}

In recent years, total hip arthroplasty through the direct anterior approach has been gradually carried out in many countries, but whether the surgical effect can reach or exceed the traditional posterolateral approach has been a hot issue debated by scholars at home and abroad. The results of this study showed that intraoperative blood loss and postoperative drainage and length of incision were significantly lower in the DAA group than in the PLA group. Compared with the PLA, in the early postoperative period, the DAA group performed better in pain scores and rehabilitation exercises, indicating that patients with DAA had less pain and better functional activity within a few days after surgery. In the third month after surgery, the DAA approach is more advantageous in terms of physical performance, walking distance, and scoring. However, in the sixth month of evaluation, there was no significant difference in the evaluation results of the two groups of approaches. This is consistent with previous reports, the main advantage of DAA is the early recovery effect. In a prospective randomized controlled trial of THA, Barrett[5] showed that patients with DAA on the first postoperative day had lower VAS scores than patients with PLA, and more people were able to walk and climb stairs freely in the sixth week after surgery. In Zhao's research[6], it was found that DAA had less muscle damage than PLA, and was considered to be responsible for better efficacy in the early stage. We chose the D'Aubigne-Postel score for joint function assessment because its scoring criteria focused more on the assessment of unilateral limb sensation and function than on the overall function of the patient like Harris, HSS or Charnley. In this way, we can more accurately evaluate the bilateral hip joints of the same patient separately. There is still some error in this scoring standard. We use more accurate gait data to make up for this deficiency. In our study, the D'Aubigne - Postel score of DAA side was higher than that of PLA at 1 st and 3rd month after surgery, but there was no significant difference at 6 th months.

Prosthesis position is also an important indicator for assessing the success of THA. For physicians who are used to lateral position THA, postural factors of lateral position DAA THA had little effect on the placement of acetabular cup and femoral stem. Poor position of prosthesis is an important factor causing instability of hip joint after operation, which affects the recovery of joint function of patients[3]. Tamaki[15] performed an average of 7.8 years of follow-up on 790 patients who underwent DAA THA. The results showed that the postoperative dislocation rate was only $0.92 \%$. Tsukada[16] and Slotkin[17] found that DAA has a lower postoperative dislocation rate, which is related to the retention of better soft tissue tension. In addition, the placement of the prosthesis can also affect postoperative dislocation. $\mathrm{Na} \varangle k a t a[18]$ reported that the position of the DAA acetabular prosthesis is more accurate. The $99 \%$ DAA acetabular prosthesises were located in the safe zone, while the PLA were $91 \%$. They believe that placing the acetabulum in the supine position will reduce the impact of pelvic tilt, which will help the surgeon to better grasp the acetabular inclination and anteversion angle. Rathod[10] and Hamilton[19] compared the differences in the inclination and anteversion angle of the DAA and PLA acetabular prostheses and found that the difference in DAA acetabular inclination and anteversion was significantly lower than that of PLA. However, in our study, the difference of acetabular inclination angle and anteversion angle between DAA and PLA is not obvious, which may be related to our research design. The operator is more accurate in grasping the acetabular anteversion angle and abduction angle of the same patient. 
There were no serious complications such as dislocation, loosening or sinking of the prosthesis at the last follow-up of all patients in this study. This indicates that the short-term clinical efficacy of the lateral position DAA THA is comparable to that of the traditional PLA THA. In the lateral position DAA THA, the hip muscles hang down due to gravity without squeezing into the incision, and the acetabulum is more fully exposed. It is also beneficial for the lower limbs to extend and adduct and the external rotation, and the proximal femur is more fully exposed. Lateral DAA does not require a special orthopedic traction bed, nor does it need to adjust the operating bed to expose the proximal femur. The operation can be performed on a common operating table, which saves the operation time.

There was no significant difference in gait recovery between patients receiving DAA THA and PLA THA 6 months after surgery. The two THA approaches were similar for postoperative functional improvement. The only important difference was that the DAA group had an advantage in lower limb activity three months after surgery. This study found that PLA abduction muscle group release and repair have an impact on functional recovery. Rathod et al found that although the PLA group had repaired the abductor muscle group and the posterior joint capsule during the operation, the difference between the two groups still existed at 1 year after surgery[10].

However, from the patient's perspective, most patients (65\%) answered that they prefer the PLA rather than DAA. Some patients have increased fear and disgust in the early stage because the DAA incision is in front, and the posterior lateral approach is more acceptable because it is invisible. From psychological stress, the patient feels more uncomfortable. The most common shortcomings of the anterior approach are long operative time, high incidence of skin complications, lateral femoral cutaneous nerve injury, and increased risk of iatrogenic fractures. In our study, the patients did not seem to have such a preference for DAA, although, in theory, they are less muscle damaged.

This study has several limitations: (1) The follow-up time was too short, only 12 months. Longer-term follow-up is needed to clarify the efficacy of both approaches; (2) The D'Aubigne - Postel score criteria are not perfect for the separate evaluation of limbs on the same patient; (3) The number of patients with bilateral THA is rare, patients with bilateral osteoarthritis are more likely to undergo THA in stages in our country(China), we need more data to study in-depth.

\section{Conclusions}

In this study, it was found that the advantage of DAA group was limited to the early postoperative period, which needs to be further confirmed. In summary, DAA THA has a better early effect in reducing pain and improving mobility. But from the patient's feeling description, it seems that the DAA is not as preferred as we expected.

\section{Abbreviations}


DAA: Direct anterior approach

PLA: Posterolateral approach

THA: Total hip arthroplasty

VAS: Visual analogy score

DDH: Developmental dysplasia of the hip

\section{Declarations}

\section{Ethics approval and consent to participate}

This study has been approved by the Ethic Committee of the Affiliated Hospital of Xuzhou Medical University (XYEY2014-xjs010-02). All patients received informed consent and signed the informed consent.

\section{Consent for publication}

Not applicable.

\section{Availability of data and materials}

All data generated or analyzed during this study are included in this published article, and the supplementary file. We do not wish to share our patients' data because it involves patient's privacy.

\section{Competing interests}

The authors declare that they have no competing interests.

\section{Funding}

The author(s) received no financial support for the research, authorship, and/or publication of this article.

\section{Authors' contributions}

SF, JNS did the study, analyzed the data, and wrote the manuscript. YuZ, YeZ, XYC, GCZ was involved in the design, data management, and analysis of the study. All authors read and approved the final manuscript.

\section{Acknowledgements}

The authors thank Yu Zhang Ye Zhang from the Department of Orthopedic Surgery, Affiliated Hospital of Xuzhou Medical University for the Working Environment for valuable technical assistance and support. 


\section{References}

1. Matt F, David O, Paul A, Edward P, Arthroplasty HPJTJo: Direct Anterior Approach Total Hip Arthroplasty: An Adjunct to an Enhanced Recovery Pathway. 2018:S088354031830603X-.

2. Post ZD, Orozco F, Diaz-Ledezma C, Hozack WJ, Ong AJJotAAoOS: Direct Anterior Approach for Total Hip Arthroplasty: Indications, Technique, and Results. 2009, 40(3):321-328.

3. Meermans G, Konan S, Das R, Volpin A, Haddad FSJB, Journal J: The direct anterior approach in total hip arthroplasty. 2017, 99-B(6):732-740.

4. Zijlstra WP, Hartog BD, Steenbergen LNV, Scheurs BW, Nelissen RGHHJAO: Effect of femoral head size and surgical approach on risk of revision for dislocation after total hip arthroplasty. 2017, 88(4):395-401.

5. Barrett WP, Turner SE, Leopold JP, \%J Journal of Arthroplasty: Prospective randomized study of direct anterior vs postero-lateral approach for total hip arthroplasty. 2013, 28(9):1634-1638.

6. Zhao HY, Kang PD, Xia YY, Shi XJ, Nie Y, Pei FXJJoA: Comparison of Early Functional Recovery After Total Hip Arthroplasty Using a Direct Anterior or Posterolateral Approach: A Randomized Controlled Trial. 2017, 32(11):S0883540317304965.

7. Zawadsky MW, Paulus MC, Murray PJ, Johansen MA, \%J Journal of Arthroplasty: Early outcome comparison between the direct anterior approach and the mini-incision posterior approach for primary total hip arthroplasty: 150 consecutive cases. 2014, 29(6):1256-1260.

8. Meneghini RM, Hozack WJJCO, Research R: Muscle Damage During MIS Total Hip Arthroplasty: Smith-Petersen versus Posterior Approach. 2006, 453(453):293-298.

9. Graves SC, Dropkin BM, Keeney BJ, Lurie JD, Tomek IMJCO, Research R: Does Surgical Approach Affect Patient-reported Function After Primary THA? 2015, 474(4):971-981.

10. Rathod PA, Orishimo KF, Kremenic IJ, Deshmukh AJ, Rodriguez JA, \%J Journal of Arthroplasty: Similar improvement in gait parameters following direct anterior \& posterior approach total hip arthroplasty. 2014, 29(6):1261-1264.

11. Radoicic D, Zec V, Elassuity WI, Azab MAJIO: Patient's perspective on direct anterior versus posterior approach total hip arthroplasty. 2018, 42(6):1-5.

12. Banaszkiewicz PA: Functional Results of Hip Arthroplasty with Acrylic Prosthesis. 2014.

13. Mayr E, Nogler M, Benedetti MG, Kessler O, Reinthaler A, Krismer M, Leardini AJCB: A prospective randomized assessment of earlier functional recovery in THA patients treated by minimally invasive direct anterior approach: A gait analysis study. 2009, 24(10):812-818.

14. Yoo JI, Cha YH, Kim KJ, Kim HY, Choy WS, Hwang SCJBMD: Gait analysis after total hip arthroplasty using direct anterior approach versus anterolateral approach: a systematic review and meta-analysis. 2019, 20(1).

15. Tamaki T, Oinuma K, Miura Y, Higashi H, Kaneyama R, Shiratsuchi HJJoA: Epidemiology of Dislocation Following Direct Anterior Total Hip Arthroplasty: A Minimum 5-Year Follow-Up Study. 2016, 31(12):2886-2888. 
16. Tsukada S, Wakui MJOOJ: Lower Dislocation Rate Following Total Hip Arthroplasty via Direct Anterior Approach than via Posterior Approach: Five-Year-Average Follow-Up Results. 2015, 9(1):157162.

17. Slotkin EM, Patel PD, Suarez JCJJoA: Accuracy of Fluoroscopic Guided Acetabular Component Positioning During Direct Anterior Total Hip Arthroplasty. 2015, 30(9):102-106.

18. Nakata K, Nishikawa MKJJoA: A clinical comparative study of the direct anterior with mini-posterior approach: two consecutive series. 2009, 24(5):698-704.

19. Hamilton WG, Parks NL, Cathy HJJoA: Comparison of Cup Alignment, Jump Distance, and Complications in Consecutive Series of Anterior Approach and Posterior Approach Total Hip Arthroplasty. 2015, 30(11):1959-1962.

\section{Figures}




\section{7 patients were eligible for this study}

15 patients refused to join in, 2 patients were lost for follow-up

20 patients were included in the study

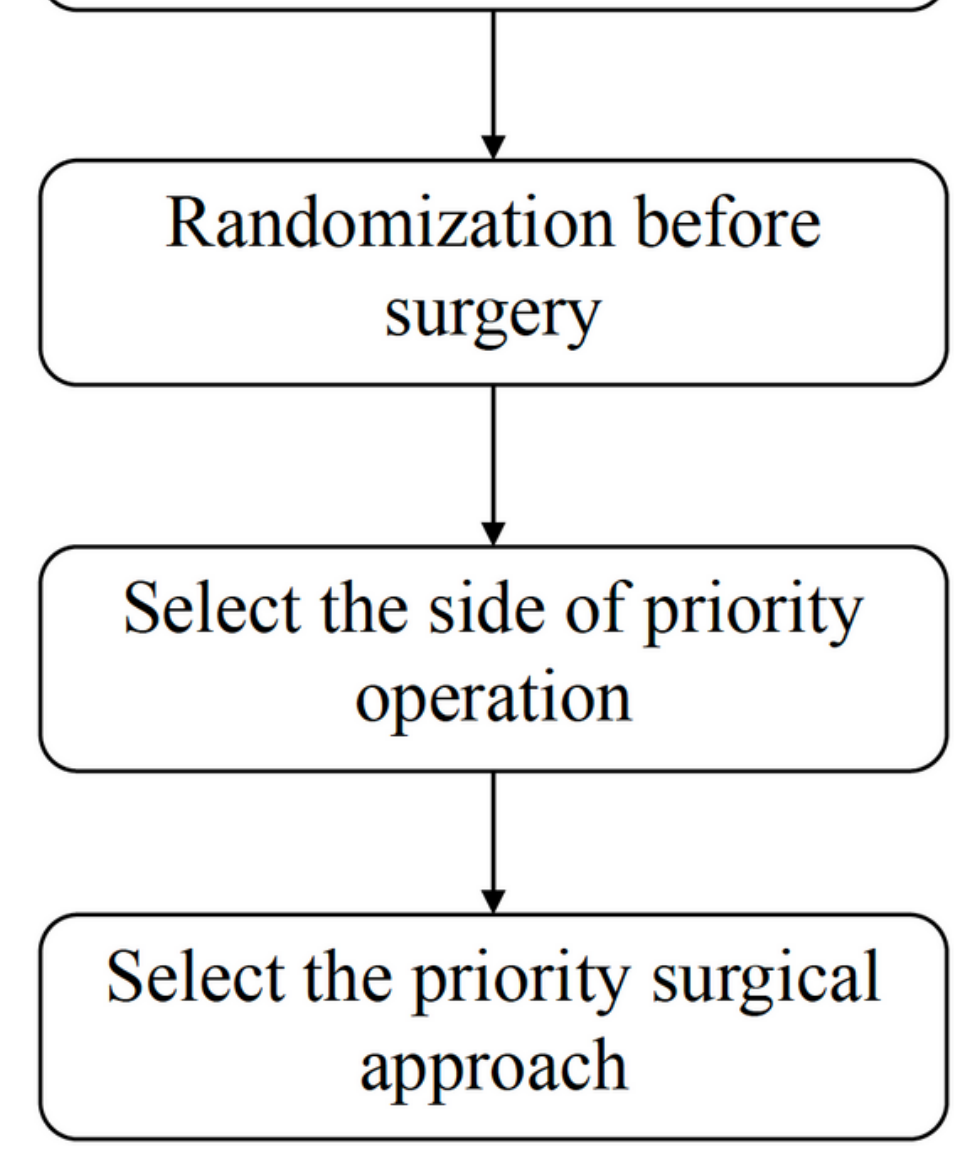

\section{Figure 1}

Flowchart depicting the patient-randomized procedure. 


\section{Acetabular position}

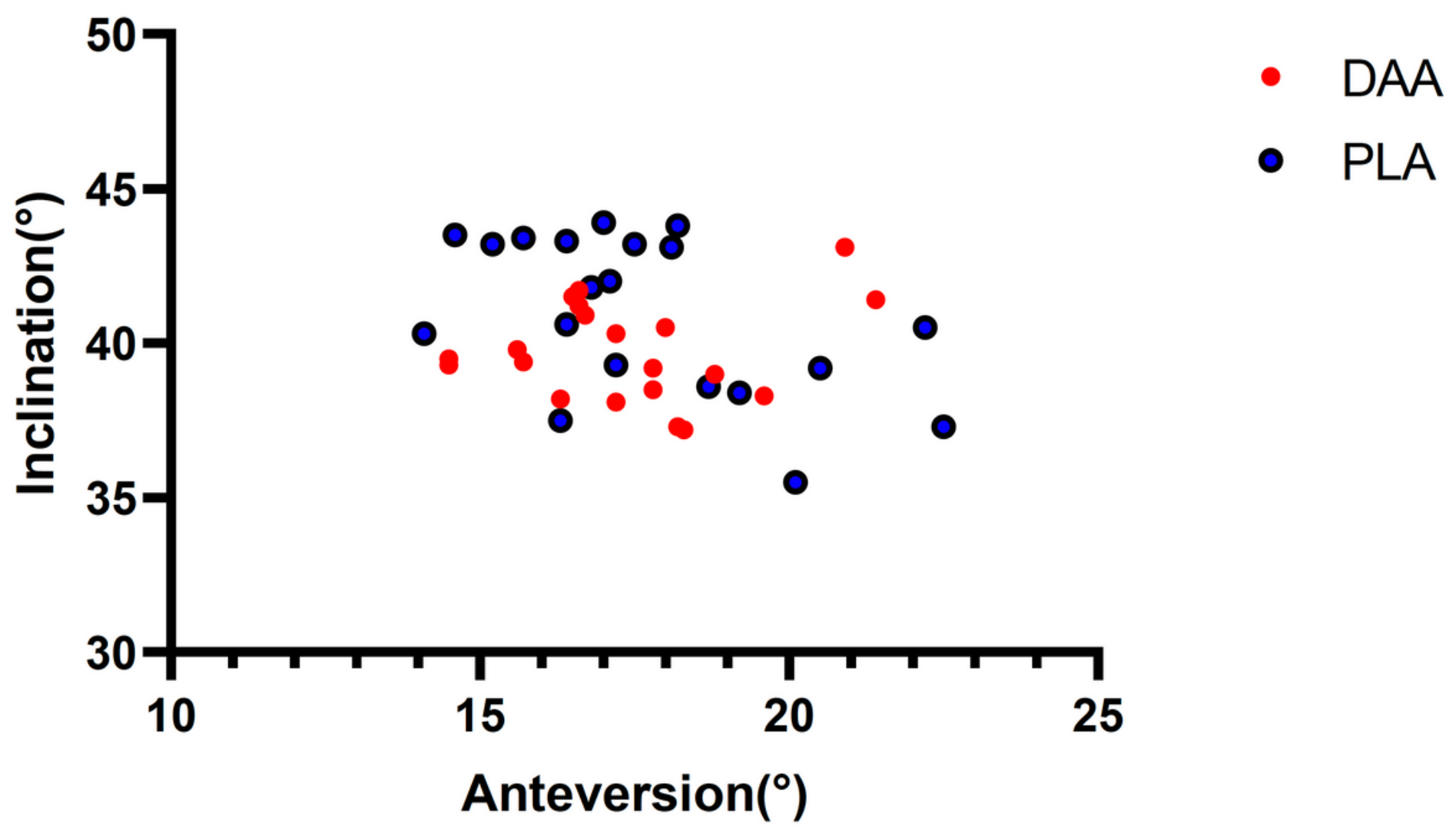

Figure 2

Scatter plot comparing variability in acetabular component placement via DAA and PLA. 


\section{Mid-Stance}

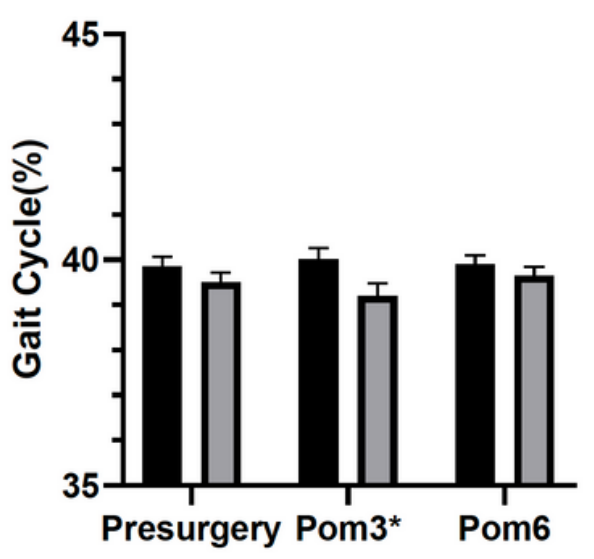

Knee Rom

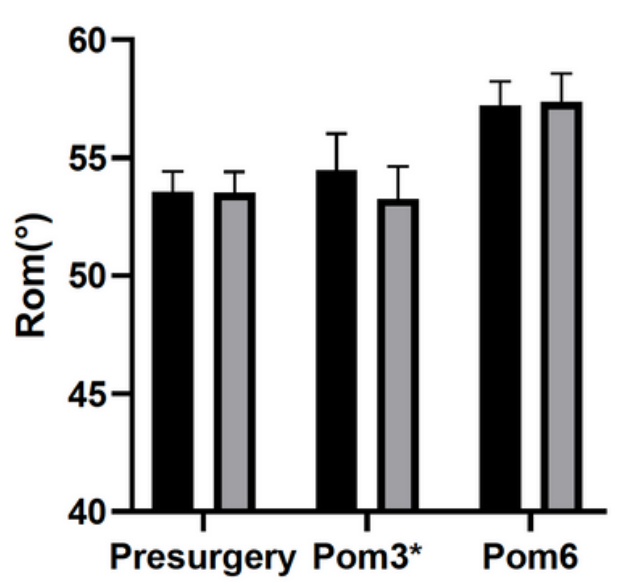

Hip Rom

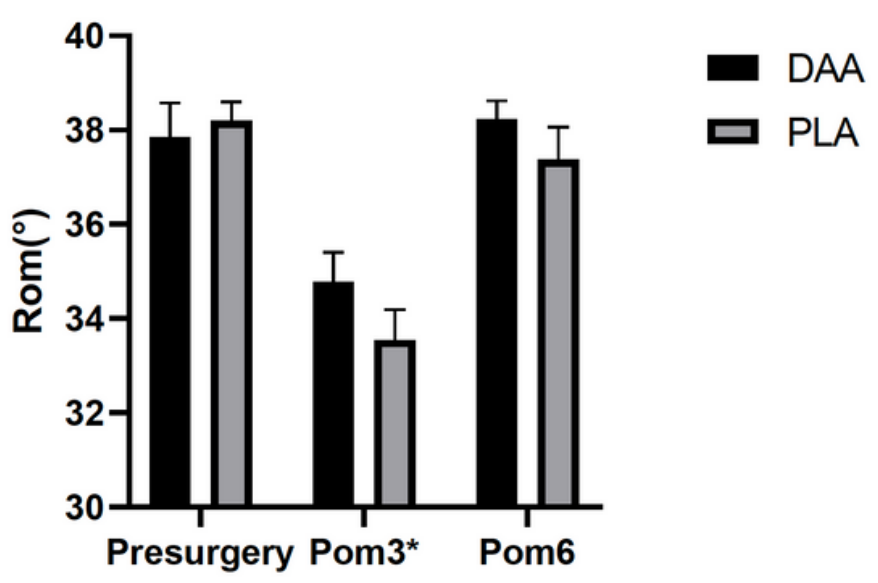

Ankle Rom

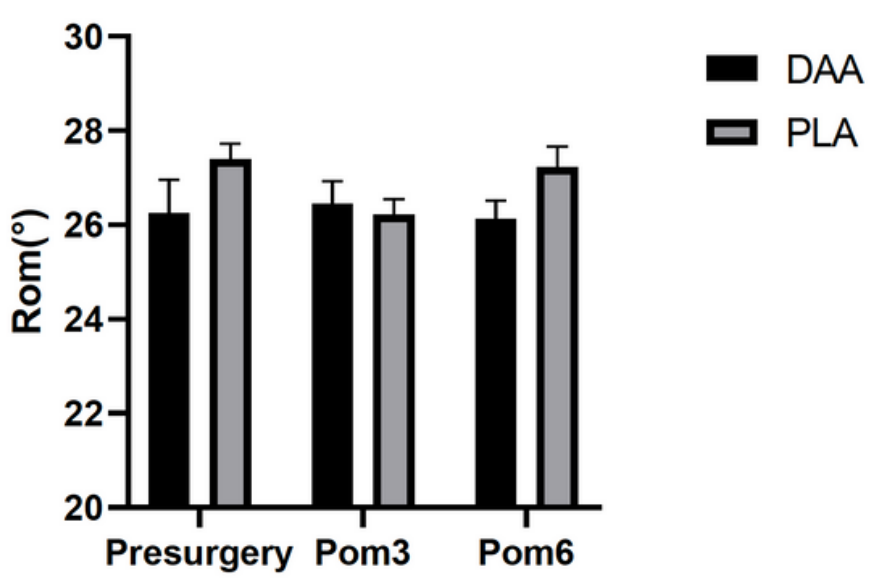

Figure 3

Gait parameters after THA via DAA or PLA, measured at Preoperative, 3 postoperative months (POM3) and 6 postoperative months (POM6). Mid-stance in gait cycle, Hip Rom, Knee Rom, Ankle Rom. * represents a statistically significant difference.

\section{Supplementary Files}

This is a list of supplementary files associated with this preprint. Click to download.

- CONSORT2010FlowDiagram1.doc

- CONSORT2010Checklist1.doc 